\title{
Three-dimensional printing flexible models: a novel technique for Nuss procedure planning of pectus excavatum repair
}

\author{
Lei Wang ${ }^{1 \#}$, Ting Guo ${ }^{1,2 \#}$, Hao Zhang ${ }^{1 \#}$, Sanhu Yang ${ }^{1}$, Jiahe Liang ${ }^{3}$, Yitong Guo ${ }^{4}$, Qiuya Shao ${ }^{5}$, \\ Tiesheng Cao ${ }^{3,4}$, Xiaofei $\mathrm{Li}^{1}$, Lijun Huang ${ }^{1}$ \\ ${ }^{1}$ Department of Thoracic Surgery, Tangdu Hospital, Fourth Military Medical University, Xi'an 710038, China; ${ }^{2}$ Department of Pain Management, \\ The Affiliated Hospital of Northwest University (Xi'an No. 3 Hospital), Xi'an 710082, China; ${ }^{3}$ Department of Ultrasound Diagnosis, Tangdu \\ Hospital, Fourth Military Medical University, Xi'an 710038, China; ${ }^{4} 3 \mathrm{D}$ Printing Research Center of the Fourth Military Medical University, Xi'an \\ 710038, China; ${ }^{5}$ Department of Urology, the First Affiliated Hospital of Xi'an Jiaotong University, Xi'an 710043, China \\ Contributions: (I) Conception and design: L Wang, L Huang; (II) Administrative support: T Cao, X Li; (III) Provision of study materials or patients: \\ T Guo, H Zhang; (IV) Collection and assembly of data: H Zhang, S Yang, J Liang; (V) Data analysis and interpretation: Y Guo, Q Shao; (VI) \\ Manuscript writing: All authors; (VII) Final approval of manuscript: All authors. \\ \#These authors contributed equally to this work. \\ Correspondence to: Lijun Huang, Xiaofei Li, Tiesheng Cao. 577\#, Xinsi Road, Xi’an 710038, China. Email: tuodi1986@msn.com; lxfchest@fmmu.edu.cn; \\ caots@fmmu.edu.cn.
}

\begin{abstract}
Background: Pectus excavatum (PE), one of the most common congenital chest wall deformities, is characterized by posterior depression of the sternum and lower costal cartilages. In this study, we demonstrated the application of flexible three-dimensional printing thoracic models for surgical approach planning of extrapleural Nuss procedure for patients with pectus excavatum.

Methods: Six patients with pectus excavatum were referred to our hospital for extrapleural Nuss procedure. Each patient's chest was reconstructed based on their computed tomography imaging data, and the threedimensional (3D) thoracic model was manufactured with flexible material using $3 \mathrm{D}$ printing technique. The individual surgical approach and custom-made steel bars were designed and produced using these models.

Results: The surgical approach was evaluated by using the three-dimensional thoracic model. In all patients received extrapleural Nuss surgery, it has been proven the uniformity of repair efficacy in both models and patients. Moreover, an individualized and well-fitting steel bar can be fabricated once the surgical approach was confirmed. All the steel bars were loaded against the ribs rigorously and seamlessly.
\end{abstract}

Conclusions: The flexible three-dimensional thoracic models were very helpful for the preoperative planning of extrapleural Nuss procedure.

Keywords: Three-dimensional printing; pectus excavatum; extrapleural Nuss procedure

Submitted Sep 14, 2019. Accepted for publication Nov 22, 2019.

doi: $10.21037 /$ atm.2019.12.124

View this article at: http://dx.doi.org/10.21037/atm.2019.12.124

\section{Introduction}

Pectus excavatum (PE), one of the most common congenital chest wall deformities, is characterized by posterior depression of the sternum and lower costal cartilages (1). In 1998, Nuss et al. (2) proposed a minimally invasive approach as an alternative to the standard open repair, involving placement of a substernal convex bar to push the sternum outward. For about 2 years, the bar remained under the sternum and was later removed surgically. The primary procedure we had previously performed was to 
enter the chest cavity without introducing a thoracoscopic extrapleural approach and allowed us to propose some modifications in the Nuss method, which were useful to reduce the irritation of the thoracic cavity and also to limit the surgical trauma $(3,4)$.

However, there were several limitations for traditional Nuss procedure. Firstly, although the advanced radiological technology, such as computed tomography (CT) and computer three-dimensional visualization, has been utilized for evaluation of chest wall deformity it was difficult to evaluate the repair efficacy of Nuss surgery, which might result in an empirical decision on the surgical approach and trajectories of the steel bar. In addition, it was difficult to assess the size of steel bar accurately because of the mobility of thorax after Nuss surgery, which might cause the displacement and looseness of the bar. To overcome the above deficiency of Nuss procedure, we developed a flexible three-dimensional (3D) thoracic model to simulate the surgery procedure and evaluate the repair efficacy. As compared with previous rigid $3 \mathrm{D}$ printings for surgery planning $(5,6)$, the flexible models can be further used to rehearse the Nuss procedure and fabricate the steel bar, which can predict accurately and intuitively the surgery efficacy.

\section{Methods}

Six patients ( 4 males and 2 females, age $4.5-7.5$ years) with symmetrical or mildly asymmetric PE were enrolled in this study. These patients were referred to our hospital for complete the non-thoracoscopic extrapleural Nuss procedure which has been described in our previous reports $(3,4)$. All patients volunteered to participate in the study and signed informed consent forms. The chest radiography (posteroanterior and lateral) and 64-slice CT were performed to evaluate the extent and nature of the deformity, and the Haller index was calculated (ratio of the transverse diameter of the chest to the anteroposterior distance, as measured from the anterior border of the vertebral body to the posterior border of the sternum). Before the surgery, we built a 3D chest model for each patient in order to better understand the surgical approach and steel bar making.

A high-accuracy 3D CT scanner (GE Lightspeed VCT, USA) with a slice thickness of $1.25 \mathrm{~mm}$ was used to obtain preoperative images of the thorax (Figure 1A). The images were exported as DICOM format to $3 \mathrm{D}$-DOCTOR imaging software (Able Software Corp, USA). The intensity threshold of costicartilage was defined from 50 to 150 Hounsfield units (HU). Reconstructed costa and costicartilage digital models were checked on several optional planes in order to confirm the structural accuracy. Before export of the data to the $3 \mathrm{D}$ printer, the digital model was smoothed to compensate for coarseness caused by the segmentation making sure that it would not change the characteristics of the thorax structures. The flexible polylactic acid materials were utilized to produce the thoracic model.

These newly 3D thoracic models allowed us to clearly identify the dimensions of the deformities and the exact localization of the safe trajectories of the steel bar. As shown in the Figure $1 B$, the funnel was paralleled with the fourth and fifth intercostal space. Although the deepest bottom of the funnel was aligned with the xiphoid and the fifth intercostal space, the xiphoid was not stable enough to support the steel bar. Whilst the equivalent repair efficacy was observed after the steel bar being respectively penetrated into the fourth and fifth intercostal space in the $3 \mathrm{D}$ thoracic models. Thus, the fourth intercostal space was confirmed as the surgical approach and trajectories of the steel bar. Furthermore, the steel bar can be fabricated according to the perimeter and radian of the $3 \mathrm{D}$ thoracic models, which was also corresponded to deformities of the patient.

\section{Results}

A total of 6 patients received the extrapleural Nuss procedure with $3 \mathrm{D}$ thoracic models guiding. Notably, the plastic model provides a possibility for predicting the outcome of surgery. Compared with rigid plastic materials, the flexible polylactic acid materials have certain elasticity and toughness. Thus, we can predict the repair efficacy using this flexible 3D thoracic model. In Figure $1 C, D$, the funnel of thorax was repaired completely after the Nuss procedure, and the same repair efficacy in $3 \mathrm{D}$ thoracic model was observed. Otherwise, an exact and personalized steel bar can be fabricated by using this $3 \mathrm{D}$ thoracic model. As shown in Figure 2, the perimeter of thorax has barely changed. The transverse diameter would decrease when 

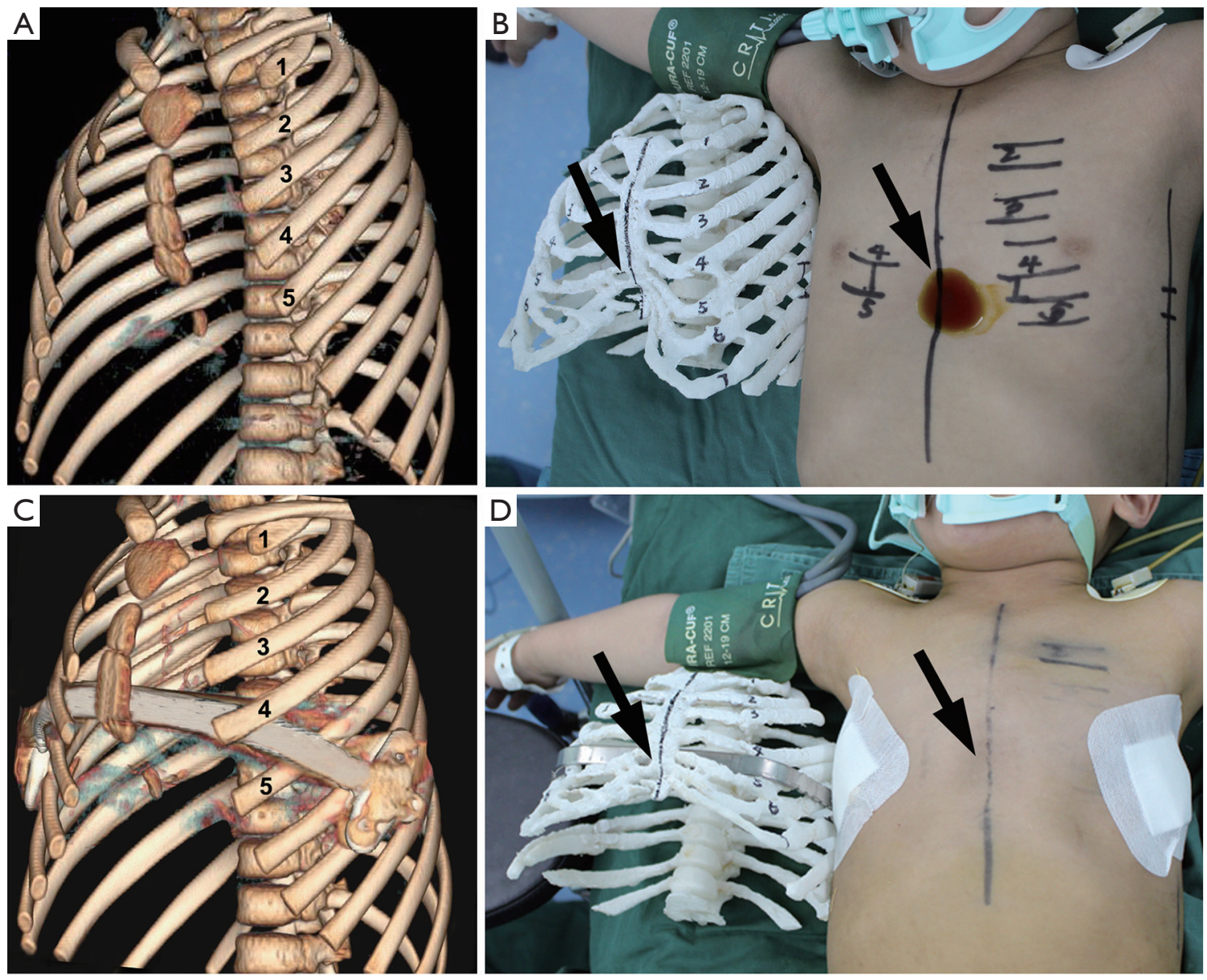

Figure 1 Validation of three dimensional printing (3DP) thoracic model in NUSS surgery. (A) Three-dimensional visualization of computed tomography of the patients before Nuss surgery; (B) the pectus excavatum (arrows) of 3DP thoracic model and the patient; (C) threedimensional visualization of computed tomography of the patients after Nuss surgery, and the steel bar was penetrated from the fourth intercostal space; (D) the substernal force point (arrows) of 3DP thoracic model and the patient.

the funnel was pushed up by the steel bar. It was obviously inaccurate to make the steel bar in accordance with the thorax of patient. Hence, the 3D thoracic model can give the exact size and radian of the steel bar. In these 6 patients, all the steel bars were loaded against the ribs rigorously and seamlessly, which can reduce the occurrence of displacement of bars and skin ulcer (Figure 3).

\section{Conclusions}

$3 \mathrm{D}$ printing technique has been frequently used for cardiovascular surgery planning (5-9). It was proven the superiority of the 3D models for surgical decision-making, especially for the complex aortic arch surgery (7-9). The thorax is different from other organs due to the mobility during breathing. The location of ribs, centrums and sternum would move after the orthopedics of thorax. In this study, we constructed a 3D thoracic model using flexible material, and we believe that it was superior to rigid plastic material for 3D thoracic model.

The extrapleural Nuss procedure was a classic surgery for repair of PE. A steel bar was penetrated from chest wall, intercostal space and anterior mediastinum, and the abnormal sternum was supported by the steel bar which was loaded against the ribs. Therefore, it must be worth noting the choice of substernal force point in surgery can 

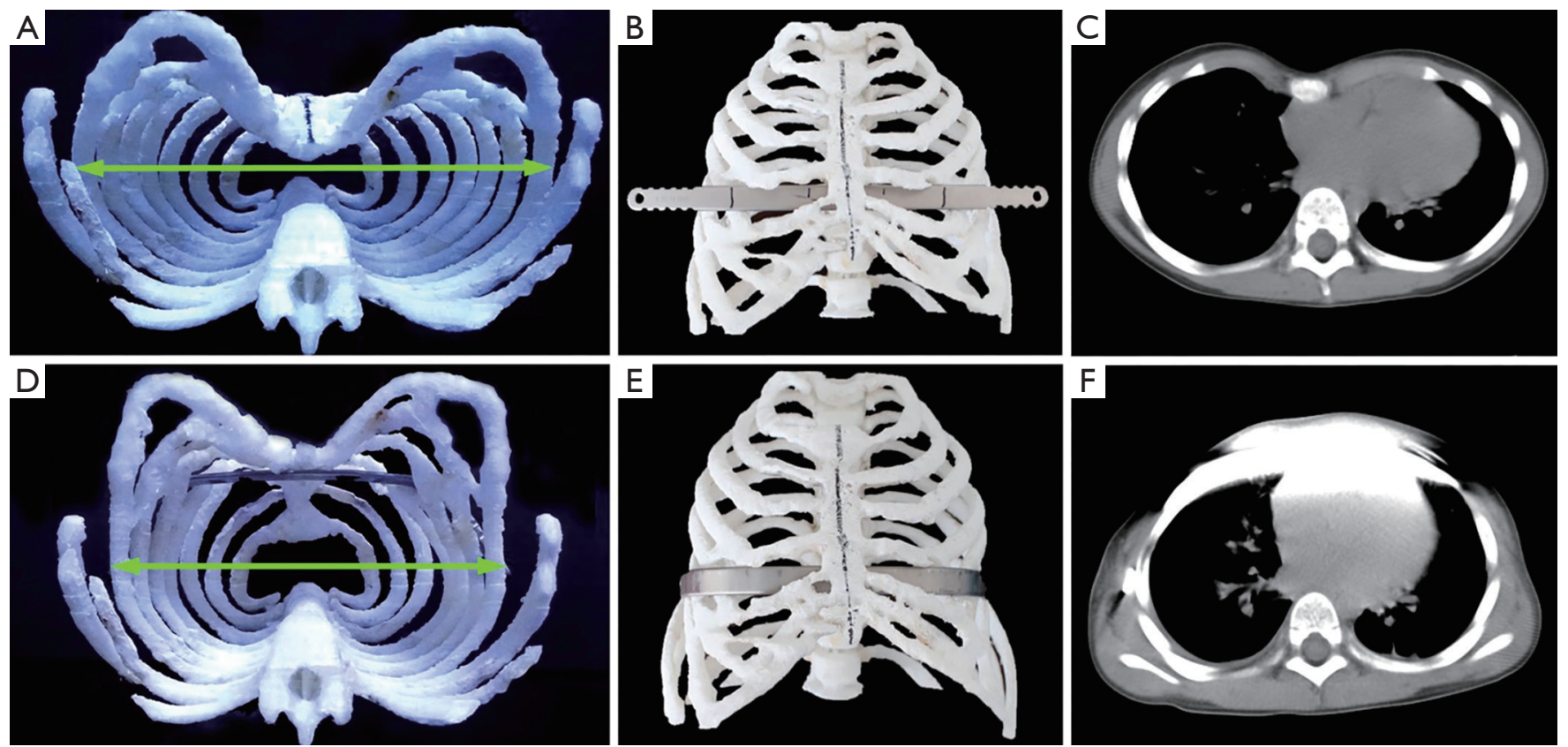

Figure 2 Application of three dimensional printing (3DP) thoracic model to simulate the NUSS surgery. (A) The transverse diameter of the thorax before Nuss surgery; (B) the initial steel bar was penetrated into the 3DP model; (C) The transverse diameter of the thorax was decreased after Nuss surgery; (D) the processed steel bar was placed into the 3DP model; (E) The mediastinal window of the chest CT images before Nuss surgery; $(\mathrm{F})$ the mediastinal window of the chest CT images after Nuss surgery.

significantly influence the repair efficacy. The optimal force point can achieve a perfect repair efficacy, as to be firm enough until the removal of steel bar. However, especially for some patients with asymmetric or severe PE, it became quite difficult to find the optimal substernal force point. The surgical approach was always determined according to the doctors` experiences, which leads to the uncertainty of repair efficacy. Therefore, a novel method should be established for the decision-making.

As a consequence, we utilized the flexible $3 \mathrm{D}$ thoracic model to find the optimal substernal force point and estimate the repair efficacy of Nuss procedure. We can penetrate the steel bar from variant intercostal space in these models to find the optimal substernal force point and surgical approach before surgery. In all 6 patients, it has been proven the uniformity of repair efficacy in both $3 \mathrm{D}$ models and patients, which showed the assistant role for the preoperative decision making and planning of extrapleural Nuss procedure. Moreover, an individual and well-fitting steel bar can be fabricated once the surgical approach was confirmed. Due to the mobility of thorax, the transverse diameter would decrease when the funnel was repaired. All the steel bars were loaded against the ribs rigorously and seamlessly. One of the difficulties with this technique was the construction of costal cartilage because it was very difficult to distinguish the soft tissue from costal cartilage in chest CT, which required adjustment of the center and width of window to enhance the differences of the soft tissue and costal cartilage.

In conclusion, the method described is feasible for preoperative decision making and planning of extrapleural Nuss procedure. The flexible 3D thoracic models can provide an exactly surgical approach and an individual and well-fitting steel bar. More data are expected to show that the use of preoperative models increased the repair efficacy and decreased the displacement of steel bar. In the future studies, a 3D titanium bar may be directly designed and printed instead of the traditional steel bar. The Nuss surgery may become more exactly depending on $3 \mathrm{D}$ printing technique. 

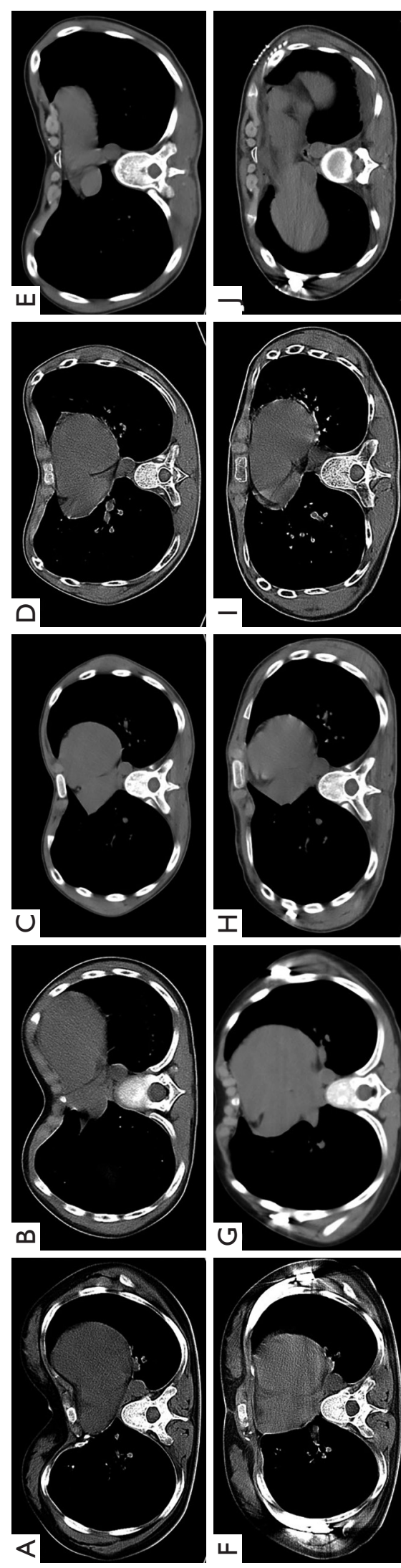

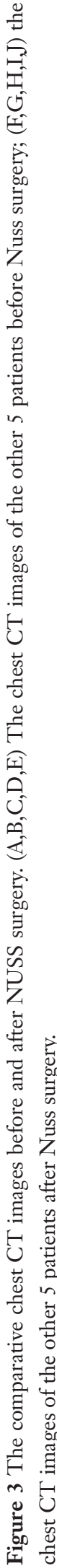

\section{Acknowledgments}

Funding: This work was supported by Key research and development plan in Shaanxi province (2019SF-095, 2018SF-056), National Natural Science Foundation of China (51835010, 81901838), Research and development program in key areas of Duangdong province (2018B090906001). This study was conducted with freedom of investigation.

\section{Footnote}

Conflicts of Interest: The authors have no conflicts of interest to declare.

Ethical Statement: The authors are accountable for all aspects of the work in ensuring that questions related to the accuracy or integrity of any part of the work are appropriately investigated and resolved. This study was approved by the ethics committee of Tangdu Hospital, the Fourth Military Medical University (Ethics approval number: TDLL-2016196-450). All patients volunteered to participate in the study and signed informed consent forms.

\section{References}

1. Brochhausen C, Turial S, Müller FK, et al. Pectus excavatum: history, hypotheses and treatment options. Interact Cardiovasc Thorac Surg 2012;14:801-6.

2. Nuss D, Kelly RE Jr, Croitoru DP, et al. A 10-year review of a minimally invasive technique for the correction of pectus excavatum. J Pediatr Surg 1998;33:545-52.

3. Han Y, Wang J, Li W, et al. Non-thoracoscopic extrapleural Nuss procedure for the correction of pectus excavatum in children. Eur J Cardiothorac Surg 2010;37:312-5.

4. Zhao J, Ma N, Yuan L, et al. Ultrasound-guided extrapleural Nuss procedure for pectus excavatum repair. Ann Thorac Surg 2014;98:1863-4.

5. Schmauss D, Schmitz C, Bigdeli AK, et al. Threedimensional printing of models for preoperative planning and simulation of transcatheter valve replacement. Ann Thorac Surg 2012;93:e31-3.

6. Schmauss D, Haeberle S, Hagl C, et al. Threedimensional printing in cardiac surgery and interventional cardiology: a single-centre experience. Eur J Cardiothorac Surg 2015;47:1044-52.

7. Schmauss D, Juchem G, Weber S, et al. Threedimensional printing for perioperative planning of complex 
Page 6 of 6

aortic arch surgery. Ann Thorac Surg 2014;97:2160-3.

8. Sodian R, Schmauss D, Schmitz C, et al. 3-dimensional printing of models to create custom-made devices for coil embolization of an anastomotic leak after aortic arch replacement. Ann Thorac Surg 2009;88:974-8.

Cite this article as: Wang L, Guo T, Zhang H, Yang S, Liang J, Guo Y, Shao Q, Cao T, Li X, Huang L. Three-dimensional printing flexible models: a novel technique for Nuss procedure planning of pectus excavatum repair. Ann Transl Med 2020;8(4):110. doi: 10.21037/atm.2019.12.124
Wang et al. Three-dimensional printing for Nuss procedure

9. Sodian R, Schmauss D, Markert M, et al. Threedimensional printing creates models for surgical planning of aortic valve replacement after previous coronary bypass grafting. Ann Thorac Surg 2008;85:2105-8. 